\title{
MIXED RADIATION FIELD DOSIMETRY UTILIZING NUCLEAR QUADRUPOLE RESONANCE
}

$\mathrm{DOE} / \mathrm{ER} / 12890--\mathrm{T} 1$

Continuation Proposal

DE92 005139

\section{TECHNICAL PROGRESS}

\section{Introduction}

This project has proposed to develop a novel dosimetry system that is capable of directly evaluating the chemical/biological damage caused by neutrons, photons, or both in a single measurement. The dosimeter itself will consist of a small volume of biological equivalent material that is probed for radiation damage with Nuclear Quadrupole Resonance (NQR) techniques. NQR has previously been utilized as a sensitive probe of structuri $l$ and chemical changes at the molecular level for a variety of organic compounds. The biological equivalent materials used in this study will not only have a density similar to tissue (tissue equivalent) but will have the same atomic components as tissue. This is a significant requirement if the important neutron interactions that occur in tissue are to occur in the dosimeter as well.

The overall objective of this studv is to investigate a methodology to perform accurate mixed-field (neutron and photon) dorimetry for biological systems. The specific objectives, as cited in the original proposal, are:

1. Choose a biological material that has an atomic composition suitable for applications as a mixed-field dosimeter, while also providing a simple NQR spectrum and strong signal strength.

2. Characterize the NQR spectral changes of the dosimete $i$ material as a function of neutron absorbed dose and energy.

3. Characterize the NQR spectral changes of the dosimeter material as a function of photon absorbed dose and energy.

4. Characterize the NQR spectral changes of the dosimeter materials in mixed radiation fields delivering known absorbed doses of neutrons and photons at known energies.

5. Optimize the characteristics of this dosimetry system for routine dosimeter applications. 
This document reports on work that was performed during the 10th - 22nd months of this project. During this time period irradiation studies have been performed on several candidate dosimeter materials to characterize the NQR response resulting from photon and neutron irradiation. The extraction of quantitative parameters describing these changes has required the development " sophisticated data analysis algorithms. Several such methodologies are now operational and are being implemented for routine data reduction in these studies.

Four students are currently performing their graduate research work on this project. Two students are Department of Energy Fellows and did not require assistantships funded from this project for the past year. Additionally one student has graduated, having completed his Masters Project entitled, "Biochemical Model Dosimetry System Based on Nuclear Quadrupole Resonance of Crystallized Hydrated Urea." This work has also been presented at the 1990 Annual Meeting of the Health Physics Society, and two scientific papers are in preparation.

\section{Selection and Evaluation of Dosimeter Materials}

The process of selecting compounds that may perform as acceptable dosimeter materials is the first objective of this project (objective \#1 from the original proposal). A number of potential dosimeter materials have been examined for their NQR and radiation responses. The initial selection of candidate dosimeter materials was based on the results of a literature review of previously performed NQR and radiation chemistry oriented experiments. The compounds chosen for initial experiments were selected on the basis of the number and type of nitrogen sites in the compound the resonance characteristics of the compound, and the various types of radiatiun damage that may be manifest in the material. Each of these materials was experimentally evaluated to determine the strength and lineshape of the $\mathrm{N}^{14} \mathrm{NQR}$ signal.

A series of samples were chosen for these studies based upon strong NQR responses and desirable compositions and structural properties. These samples are included in the family of urea derivatives, i.e. each is based on basic molecular units similar to that of urea. The specific materials chosen include:

\section{Urea}

\section{Thiourea}

\section{Guanidine Carbonate}

\section{Guanidine Sulfate}

Schematics of the chemical structures of these compounds are illustrated in figure 1. Urea provires the simplest molecular form of these compounds (figure 1a) and contains two equivaler.c nitrogen sites, resulting in a strong NQR signal and a simple spectral response. 
a. UREA

$$
\mathrm{H}_{2} \mathrm{~N}-\mathrm{C}-\mathrm{NH}_{2}
$$

b. THIOUREA

$$
\mathrm{H}_{2} \mathrm{~N}-\underset{S}{\mathrm{C}}-\mathrm{NH}_{2}
$$

c. GUANIDINE CARBONATE

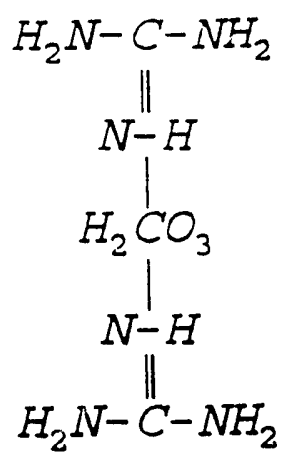

Figure 1 
Thiourea also has a simple molecular structure that is very similar to that of urea (figure 1b). The only difference in composition between thiourea and urea is that the oxygen atom in urea is replaced by a sulfur atom in thiourea. This change, however, causes the two nitrogen sites to become slightly inequivalent, so that they result in nitrogen resonances at two different frequencies. The resonant frequencies are close enough together that they may be observed simultaneously using pulsed NQR techniques, but must then be deconvoluted to quantify the contributions from each site.

Guanidine carbonate and guanidine sulfate have more complex molecular structures consisting of multiple urea-like molecular units connected by carbonate or sulfate groups, respectively (figure 1c). These materials contain six nitrogen sites, which are typically all inequivalent. In most cases the resonance frequencies are in close proximity and may all be observed simultaneously using pulsed NQR techniques. This leads to the more difficult problem of deconvolving six individual signals from the NQR response in order to perform a quantitatively meaningful interpretation.

This selection of compounds was made in order to take advantage of the similarities in the structural units of each of the compounds while permitting a variation in the level of complexity of the associated bonding structures for different compounds. It is expected that the more complex compounds will exhibit greater sensitivity to radiation induced changes because of the greater opportunity for bonding rearrangements. In order to fully understand these changes, however, it is also necessary to identify the changes that can result in the fundamental molecular units of the compounds, necessitating detailed studies of simple compounds such as urea and thiourea.

\section{Data Analysis Techniques}

In order to accurately quantify the spin-spin relaxation times characterizing the Free Induction Decays on these materials, several advanced data analysis techniques had to be developed. These include multiple site curve fitting algorithms, Fourier transform techniques, and autocorrelation algorithms.

In order to quantify spin-spin relaxation tirnes of the Free Induction Decay, nonlinear curve fitting programs were developed. Figure 2 illustrates how the two simple Free Induction Decays (the simple exponentially damped waveforms) from different nitrogen sites combine to produce a complex Free Induction Decay. Non-linear curve fitting algorithms permit the accurate determination of the spin-spin relaxation times as well as the relative weighting of the individual nitrogen sites contributing to the signal for up to three separate sites. For greater than three sites it is very unlikely that a non-linear curve fitting algorithm will converge on a unique solution. These techniques have proven to be most useful for the analysis of urea and thiourea samples, which have one and two nitrogen sites, respectively.

For sample compounds containing a greater number of sites more elaborate data analysis techniques are required. In order to separate the contributions from the individual sites, the Free Induction Decay is transformed from the time domain, to the frequency 

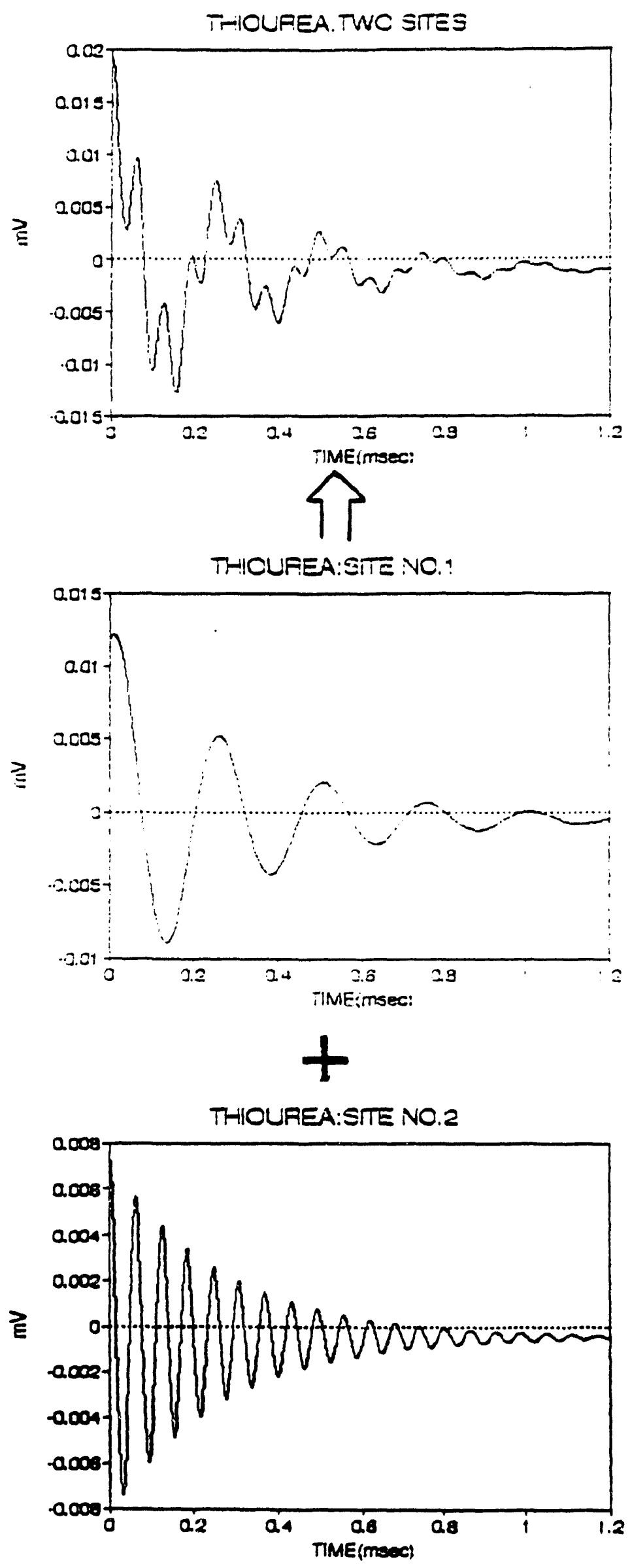

Figure 2. 
domain via Fourier transform techniques. This permits the separation of individual resonant frequencies and consequently the contributions from individual nitrogen sites. Because information of the phase of the signal is lost during data acquisition, it must be re-supplied in order to obtain meaningful interpretations from the Fourier transformed data. This requires an additional, and time consuming, step in data analysis. Analyses requiring Fourier transforms are consequently very computational and time intensive operations, but necessary at this point of the research in order to separate contributions from physically different nitrogen sites in guanidine sulfate and guanidine carbonate samples.

Autocorrelation algorithms have also been developed in order to maximize the signal/noise ratios on collected data prior to further data analysis. These algorithms have been utilized in conjunction with Fourier transform methods in order to maximize the information capable of being extracted during data analysis. The ionizing radiation may induce only small changes in frequency from the frequency of the undamaged material. The Fourier Transform cannot discern between the frequencies because of its poor frequency resolution. Therefore, capabilities have been developed to use Autoregressive Analysis. The Autoregressive method has very high frequency resolution and can easily distinguish any slight change in frequency.

It was originally anticipated that most data analysis in this project would involve straightforward quantification of NQR parameters directly from the Free Induction Decay. While this can be done for the simple spectra from urea, other candidate dosimeter materials require more sophisticated dirta analysis iechniques. We have consequently spent a greater effort on the development of data analysis techniques than originally anticipated. Currently, the vast majority of tools required for detailed analysis of the NQR signals have been developed and are being used in routine data analysis.

\section{Irradiation Experiments on Dosimeter Materials}

In order to examine the effects oi radiation that are similar to those that may be manifest in biological materials it is expected that the sample material should not only be biologically equivalent, but should have as nearly the same chemical composition as biological tissue as possible. Since a major component of all biological systems is water, it is not sufficient to have only a biological based material, such as a crystalline amino acid, present, but water molecules must also be incorporated into the material. These water molecules permit the important contributions of chemical damage that occur through the hydrolysis of water to manifest themselves in the dosimeter.

The response of hydrated samples to irradiation may therefore be expected to be very different from that of the dry, polycrystalline sample of those same materials. This has been demonstrated in all materials that irradiation studies have been initiated for. Urea, thiourea and guanididine sulfate exhibited very little response to radiation, as expected, when irradiated and observed in a dry polycrystalline form. Each, however, exhibited markedly different behavior when similar studies were performed with hydrated samples of the same compounds. 


\section{Urea}

Irradiation of hydrated urea has provided clear evidence of the radiation response for this material. Because urea is not very hygroscopic, a somewhat complex procedure was developed to incorporate water into the network. Following the addition of $4 \mathrm{ml}$ of water to a $10 \mathrm{gm}$ sample of urea, the sample was heared to the melting point and then cooled to recrystallize the hydrated sample, with water incorporated into the lattice network.

These samples served as the basis for irradiation studies of hydrated urea. Figure 3 illustrates the response of the urea NQR signal following exposure to Co- 60 gamma rays. As illustrated, the decay constant of the Free Induction Decay, the spin-spin relaxation time $\left(\mathrm{T}_{2}\right)$, exhibits a strong dependence on the delivered gamma dose. The spin-spin relaxation time decreases roughly exponentially with increasing radiation dose, providing a good correlation between the spin-spin relaxation time and the radiation dose. The delivered doses are high compared to those for personnel exposures in order to clearly demonstrate the radiation dependent behavior. Optimization techniques (discussed later) may increase the sensitivity of these techniques for practical personnel dosimetry applications. The precise mechanisms governing these changes are not yet understood but are under investigation.

Hydrated urea samples have also been examined for their response to neutron irradiation. Neutron irradiations were performed in the core of the University of Florica Training Reactor. While this exposure technique provides a mixed neutron-photon field, the neutron dose is approximately three orders of magnitude greater than the photon dose, which results in the vast majority of radiation induced changes expected for this irradiation configuration. Neutron irradiated urea exhibits a qualitatively different behavior from samples subjected to photon irradiation. Following neutron irradiation, the spin-spin relaxation time remains constant but the overall amplitude of the Free Induction Decay is greatly decreased (figure 4). Although this data is preliminary in nature, having only a few data points, it demonstrates that a marked change in the NQR response of these samples may be observed as a result of neutron irradiation. Furthermore, the qualitatively different response to neutron irradiation provides an opportunity for the simple separation of photon and neutron contributions to the absorbed dose in these materials.

\section{Thiourea}

Thiourea has two non-equivalent nitrogen sites resulting in a more complex Free Induction Decay. Quantification of these sites has been performed both through Fourier transform analysis and non-linear curve fitting. Consistent results are achieved utilizing either method, although the non-linear curve fitting methods used to extract the relative contribution to individual sites is the faster and more efficient method at this time. Irradiation studies have been performed on both polycrystalline and hydrated samples of thiourea, with the bulk of the work being performed on polycrystalline materials. Neutron irradiation studies of thiourea also demonstrate a decrease in the overall spin-spin relaxation time of the Free Induction Decay. Figure 5 illustrates how the composite Free Induction 


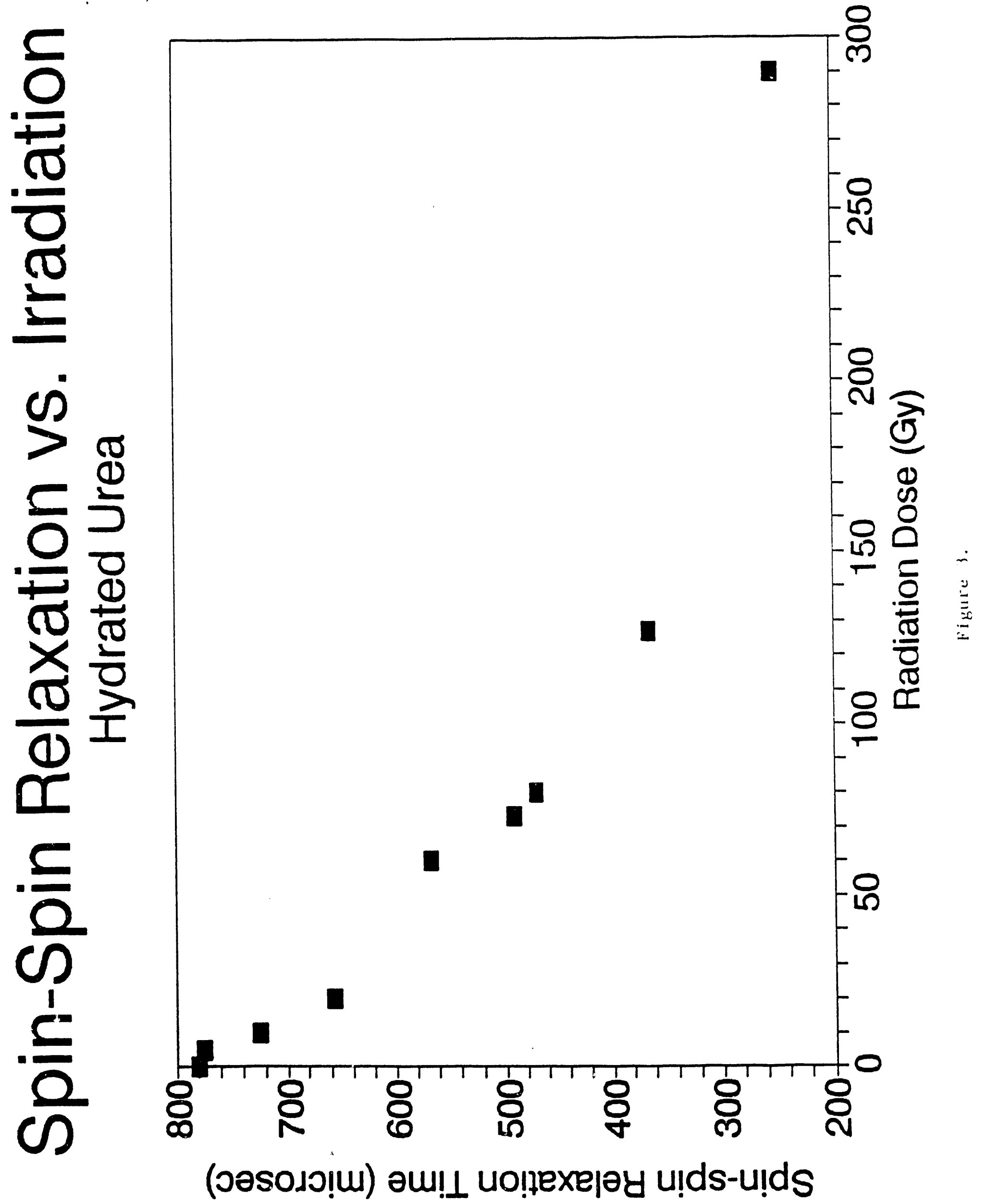




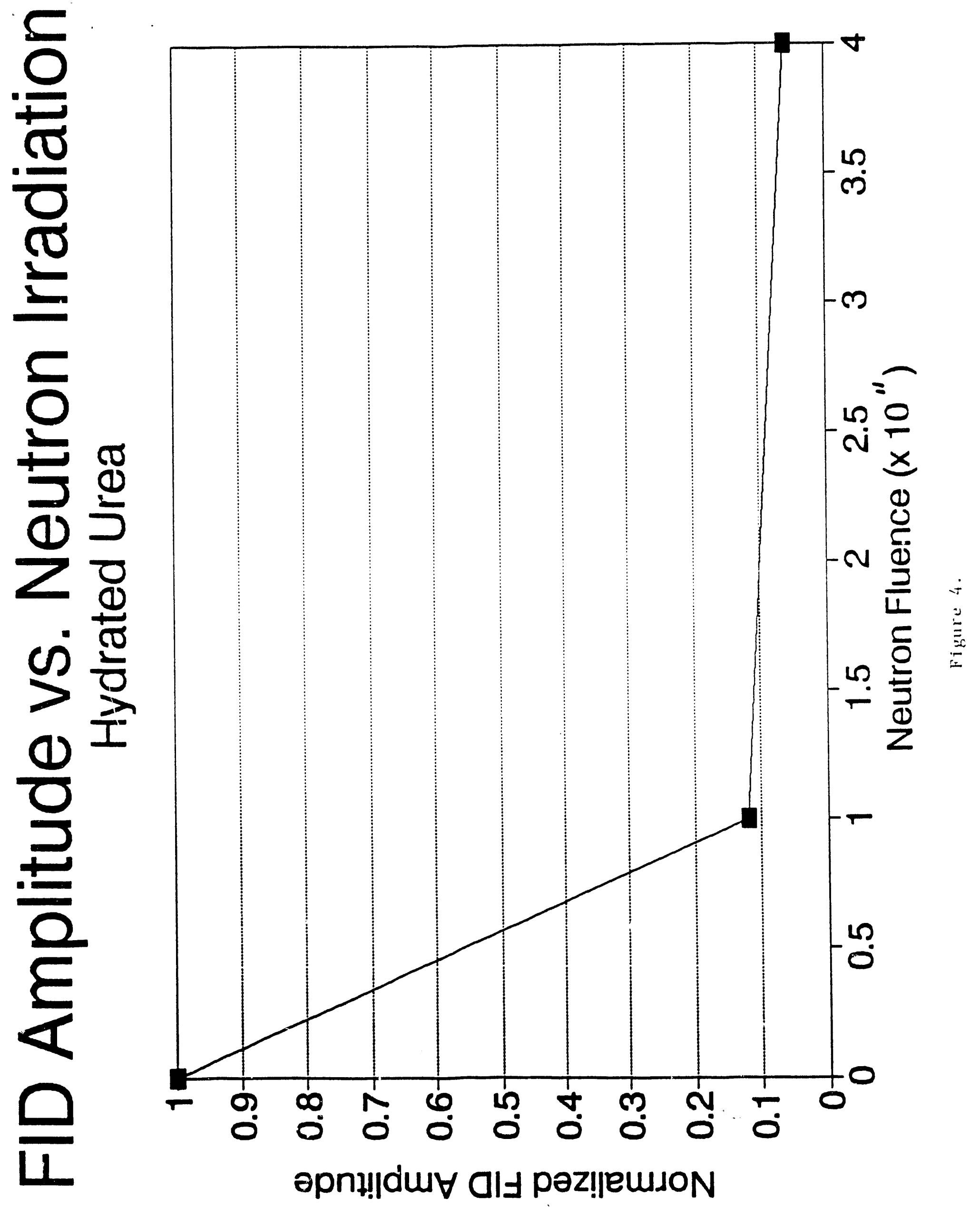


THIOLFE
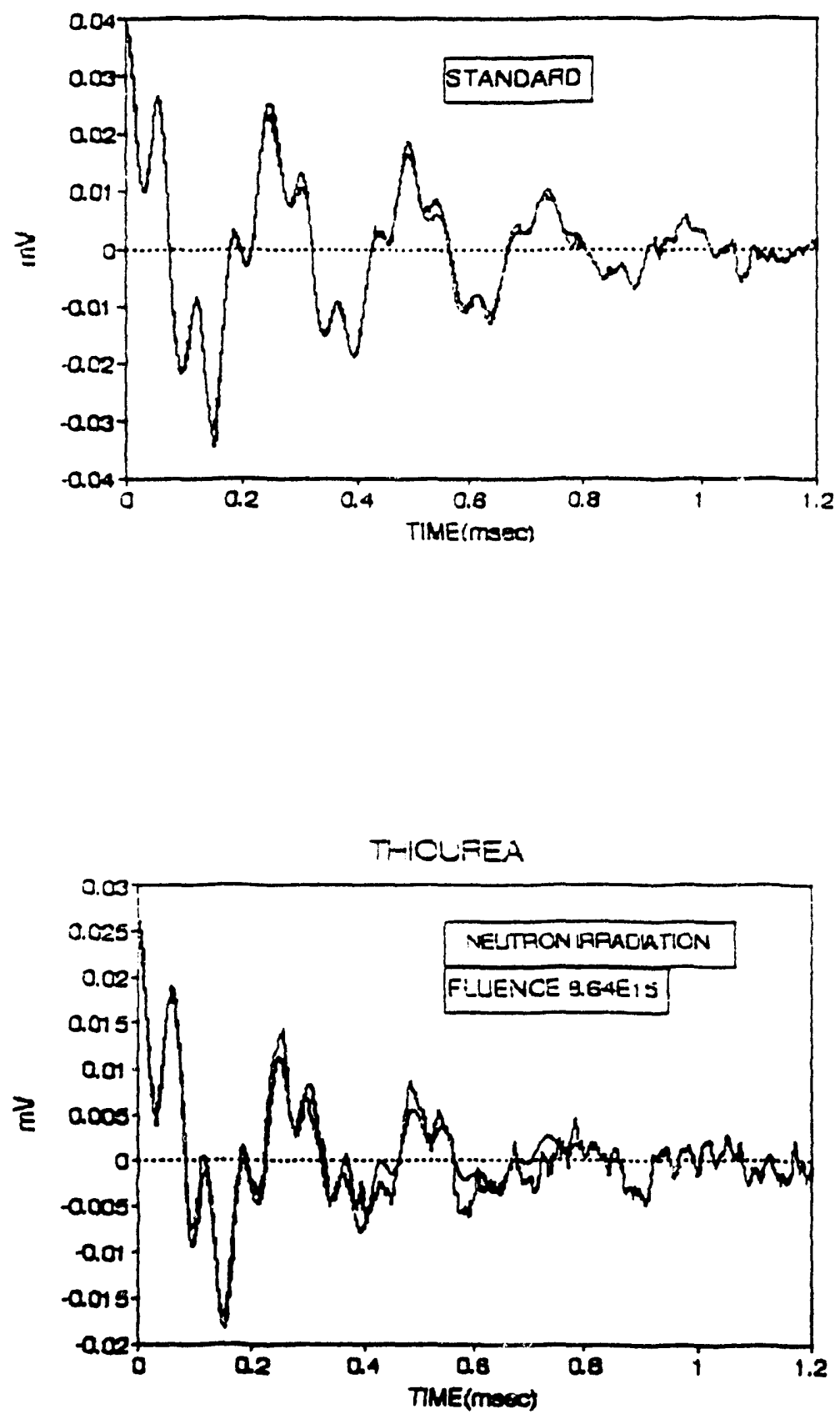
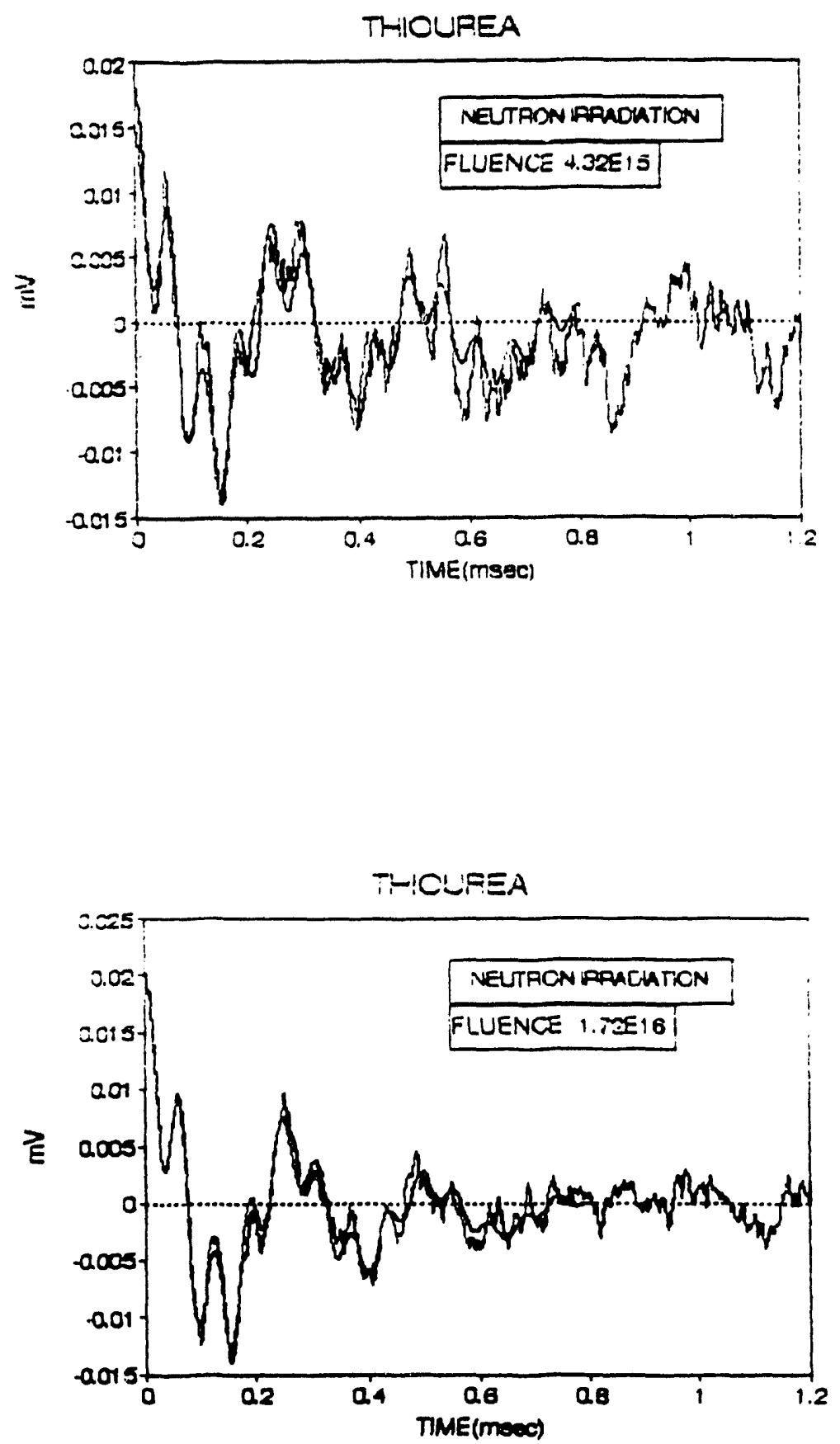

F1gure 5. 
Decay is changed with neutron irradiation. Performing a non-linear curve fit to each of these data sets (the smooth curve following the data) permit the quantification of the contributions to the Free Induction Decay from the two individual nitrogen sites.

Figure 6 demonstrates that the relaxation time for each of the nitrogen sites decreases with neutron irradiation, although site number 1 appears to be the more sensitive site.

Samples of thiourea exposed to Co- 60 gamma rays have been observed to exhibit a shortening of the spin-spin relaxation time qualitatively similar to that observed in urea. In the case of thiourea, however, the shortened $\mathrm{T}_{2}$ appears to have a time-dependent behavior and recovers over a period of several weeks. This behavior is being studied in greater detail in thiourea, and may also be able to be observed in urea samples over appropriate time periods. Successful analysis of the physical progress of radiation damage recovery in these materials could have important implications for radiation protection and risk analysis for biological systems.

\section{Guanidine Compounds}

Guanidine sulfate and carbonate have more complex NQR spectra than the other compounds discussed thus far. Guanidine sulfate, in particular, has six resonant frequencies that may all contribute to the NQR signal. The response from the respective nitrogen sites must be separated in order to evaluate the response of the samples to irradiation. In order to observe the contributions from individual sites for these materials the Free Induction Decay is Fourier transformed into the frequency domain. The complex response from the Free Induction Decay, as illusirated in figure 7 , is separated into individual trequency components demonstrated in figure 8 . Once these sites are deconvolved the variations in relative site populations and line broadening as a function of irradiation may be deternined. This data analysis procedure is currently underway for a series of experiments that have been performed on guanidine sulfate.

\section{PLANS FOR THE COMING YEAR}

Research plans for the coming year are expected to follow along the guidelines presented in the original proposal. That proposal provided for two major phases for the project, Phase I -characterization of dosimeter materials and NQR spectral responses as a function of irradiation and Phase II - the refinement and optimization of the dosimeter response. To this end, portions of Phase I and Phase II of the project will be conducted in parallel on different materials.

During the third year of funding we expect to be complete the Phase I studies and analysis on dosimeter materials that have more complex molecular structures. Irradiation studies which have been initiated will continue in order to complete the characterization of the dosimeter materials exposed to photon, neutron, and mixed irradiation fields. Analysis of this data will be geared towards developing an understanding of the mechanisms resulting in the observed NQR changes and to facilitate the eventual optimization of the dosimeter 

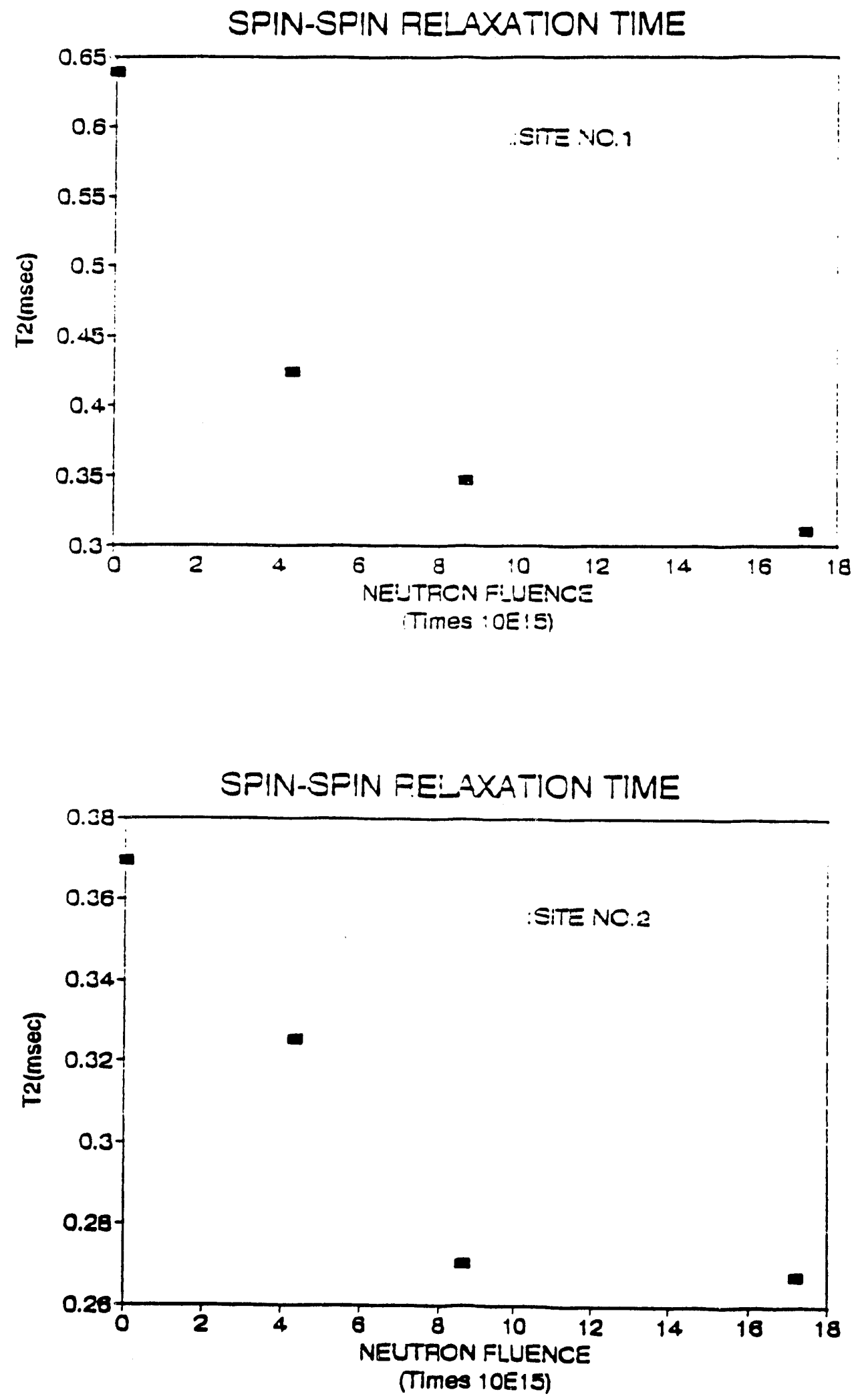


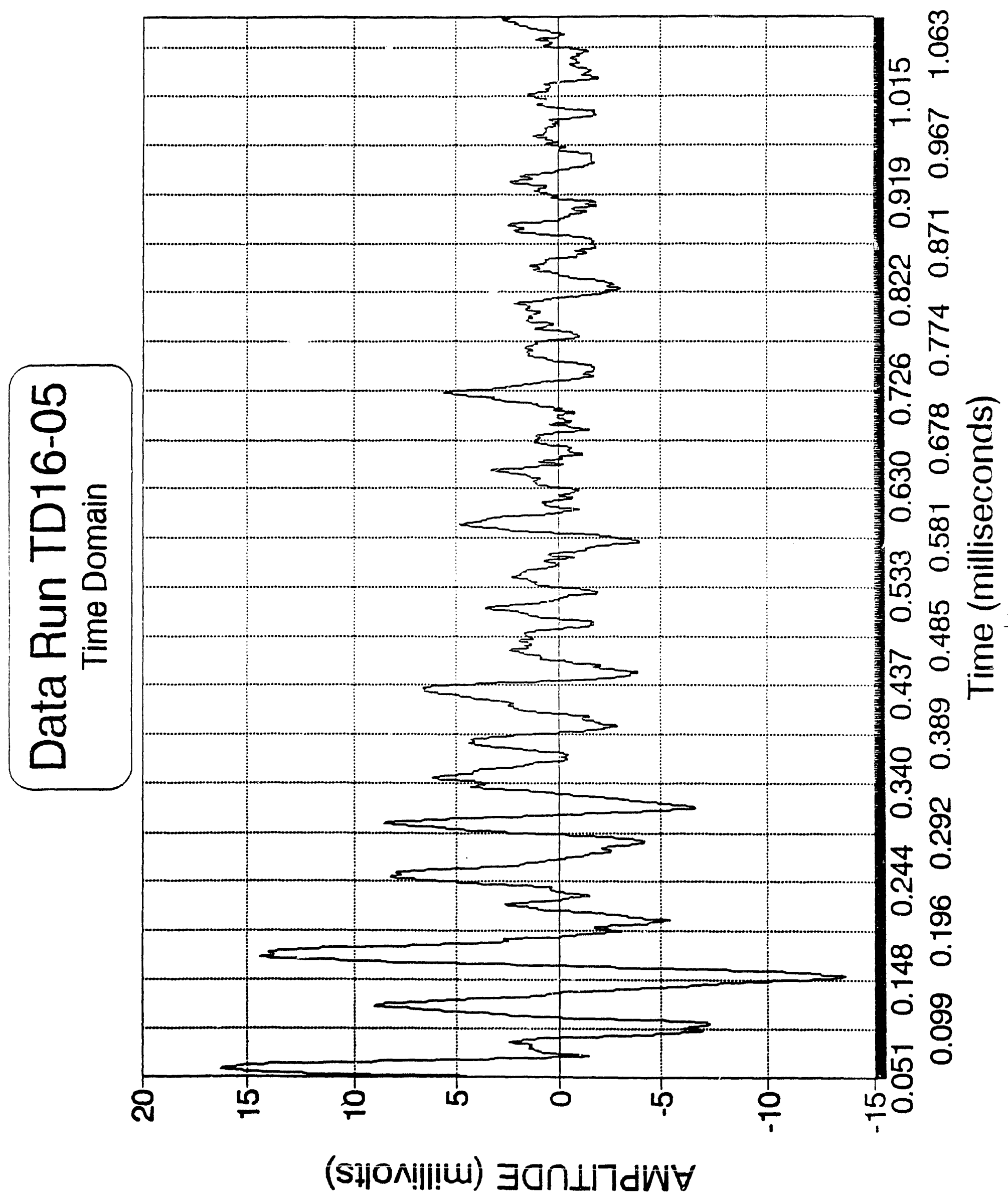




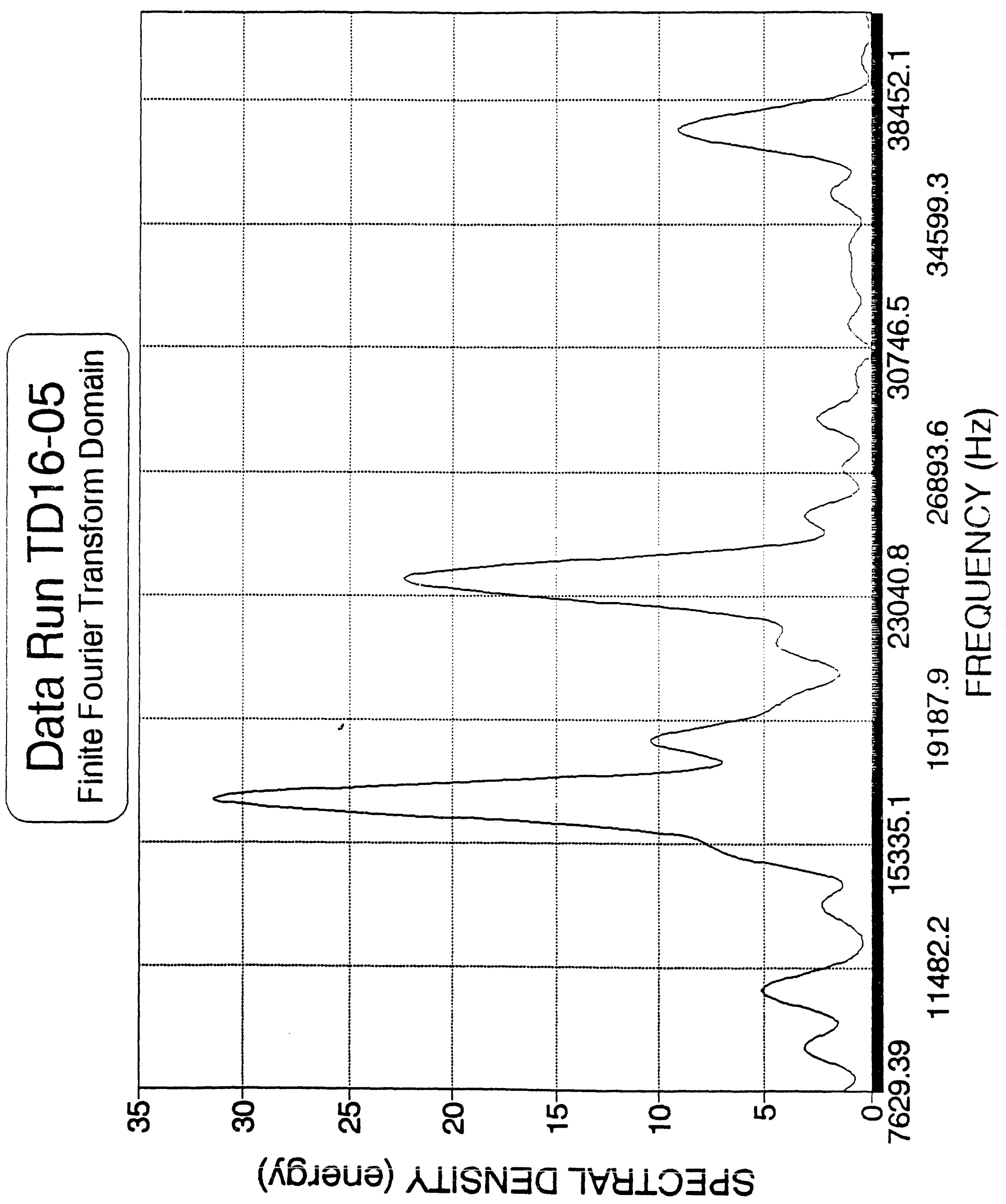


response. A complete understanding of the physical piocesses that occur in these materials will not only provide dosimeter optimization, but may have important consequences for understanding interactions of radiation in biological systems and the development of radiation risk models.

The refinement and optimization of dosimeter response will utilize several approaches. Once an understanding of the physical processes is characterized it should be possible to modify dosimeter compositions to maximize the irradiation response. Advanced data acquisition and analysis techniques will also permit a much greater level of resolution in order to provide accurate quantification at significantly lower doses than are being characterized now. Strategies under consideration for the refinement of the dosimeter materials and NQR techniques include:

1) Variation and optimization of the water content of dosimeter samples.

2) Application of a small DC magnetic field to enhance the NQR signals.

3) Utilization of more complex rf pulse sequences to maximize the response of important NQR parameters.

4) Utilization of NQR double resonance techniques.

5) Adaptation of the NQR spectrometer to a newly developed Robinson-type spectrometer.

While not all of these techniques will necessarily need to be utilized, they demonstrate that there are a number of techniques that can be followed in order to optimize this dosimetry system. These studies have demonstrated the viability of NQR tcchniques for providing quantitative measires of radiation exposure to biochemical materials for both photon and neutron radiations. It remains to develop a more complete understanding of the physical mechanisms creating these changes and developing methods for the optimization of the system for practical dosimetry applications.

\section{DISCLAIMER}

\footnotetext{
This report was prepared as an account of work sponsored by an agency of the United States Government. Neither the United States Government nor any agency thereof, nor any of their employees, makes any warranty, express or implied, or assumes any legal liability or responsibility for the accuracy, completeness, or usefulness of any information, apparatus, product, or process disclosed, or represents that its use would not infringe privately owned rights. Reference herein to any specific commercial product, process, or service by trade name, tr.Jemark, manufacturer, or otherwise does not necessarily constitute or imply its endorseme $t$, recommendation, or favoring by the United States Government or any agency thereof. The views and opinions of authors expressed herein do not necessarily state or reflect those of the United States Government or any agency thereof.
} 

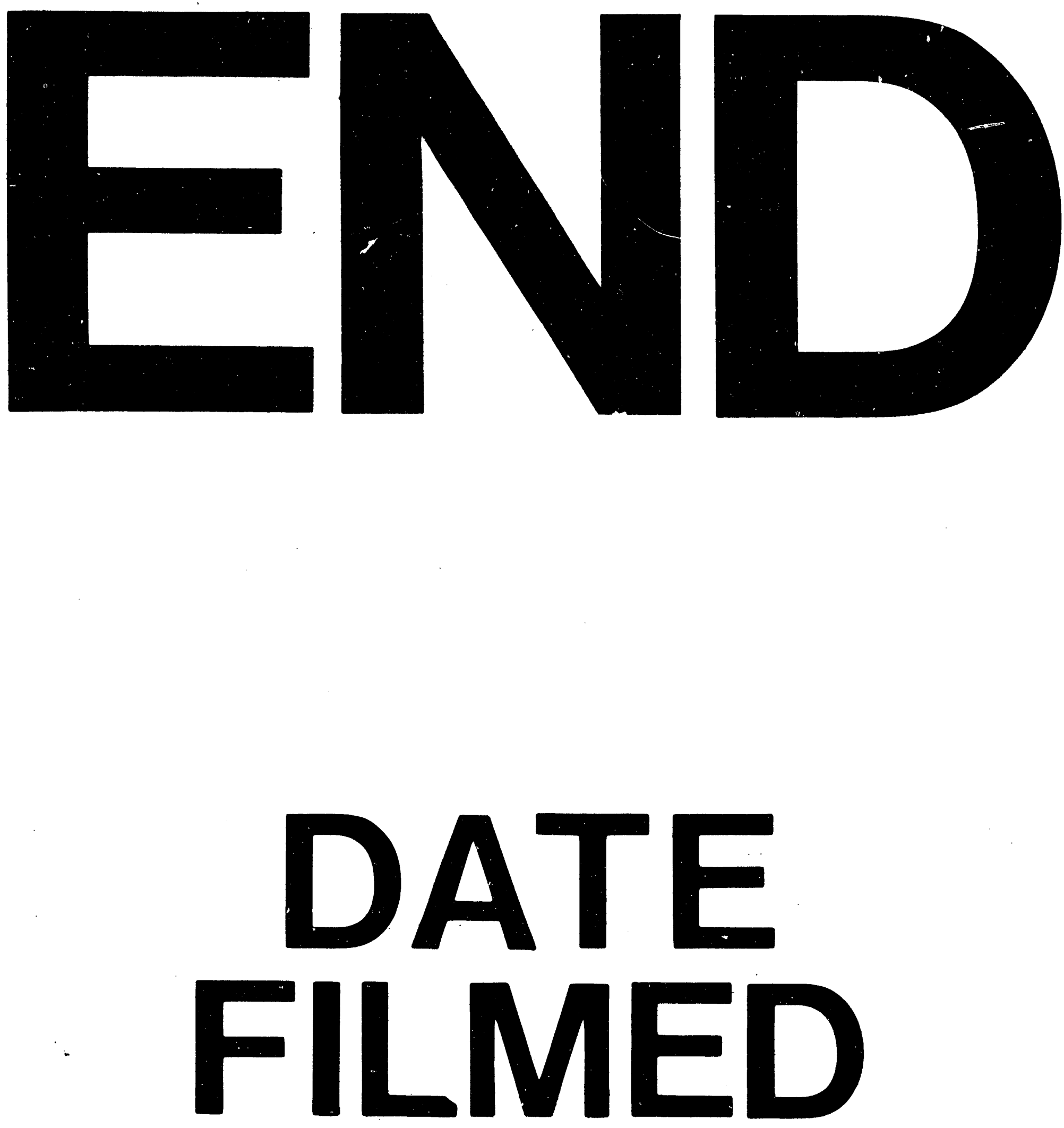

7

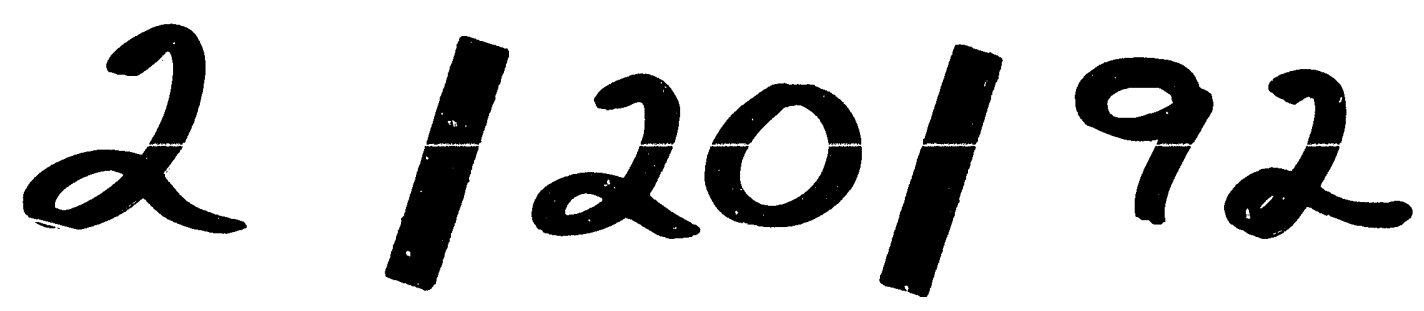


